\title{
Association of Streptococcus with Plaque Type of Psoriasis
}

\author{
Mohammad Akram Hossain ${ }^{1}$, Lubna Khondker ${ }^{2}$ \\ Received: September 18, 2014 Accepted: April 15, 2015 \\ doi: http://dx.doi.org/10.3329/jemc.v5i2.23383
}

\begin{abstract}
Background: Guttate psoriasis has a well-known association with streptococcal throat infections, but the effects of these infections in patients with chronic plaque type of psoriasis remains to be evaluated. In Bangladesh several studies were done on psoriasis but no data about association between streptococcal throat infection and plaque type psoriasis are available so far. Considering the co-morbidities of psoriasis patients, it might be justifiable to find out the events that provoke the initiation or exacerbation of psoriatic disease process. Objective: To observe the association of streptococcus with plaque type of psoriasis. Materials and Methods: This observational study was conducted in the department of Dermatology and Venereology, Bangabandhu Sheikh Mujib Medical University, Dhaka. Forty seven patients clinically and histopathologically diagnosed as having plaque psoriasis were selected as cases and patients with skin diseases other than psoriasis were selected as controls. Results: In this study majority of subjects (55\%) were diagnosed as chronic plaque psoriasis. Among the subjects with guttate flare of chronic plaque psoriasis $64.2 \%$ gave a positive history of sore throat. ASO titer was raised (>200 IU/mL) in $28(59.5 \%)$ patients of chronic plaque psoriasis and 7 (17.9\%) patients of non-psoriatic respondents. The difference between two groups was significant $(p<0.05)$. Streptococcus pyogenes was found in $12(25.5 \%)$ in chronic plaque psoriasis patients versus in $4(10.2 \%)$ in controls $(p>0.05)$. Conclusion: This study shows that streptococcal throat infections are associated with plaque psoriasis and early treatment of throat infections may be beneficial for plaque type of psoriasis patients.
\end{abstract}

Key words: Streptococcal throat infections; Chronic plaque psoriasis; Guttate flare of chronic plaque psoriasis

J Enam Med Col 2015; 5(2): 99-103

\section{Introduction}

Psoriasis is a common, chronic, inflammatory condition of the skin, characterized by red, scaly, sharply demarcated, indurated plaques present particularly over extensor surfaces and scalp. ${ }^{1}$ Psoriasis is universal in occurrence. However, its prevalence in different populations varies from $0.1 \%$ to $11.85 \% .^{2,3}$ The etiopathogenesis of the disease is still largely unknown; but studies indicate that it is caused by an interaction of multiple genetic components and environmental factors including b-hemolytic streptococci. ${ }^{4}$ There is evidence that an immunologic mechanism is involved in the triggering of psoriasis by streptococcal infections. ${ }^{5}$ Streptococcal antigens are presented by the major histocompatibility complex class II molecule on the antigen presenting cells. These lead to polyclonal $\mathrm{T}$ cell activation with release of immune cytokines, such as IL-2, which are important in the pathogenesis of psoriasis. ${ }^{6}$ Serum levels of IL-22 correlate with psoriasis severity and both IL-17 and IL-22 can induce production of antimicrobial peptides commonly seen in psoriatic skin. ${ }^{7}$ Increased numbers of interferon producing Th1 cells with specificity for group A

1. Assistant Professor, Department of Dermatology and Venereology, Sir Salimullah Medical College \& Mitford Hospital, Dhaka 2. Assistant Professor, Department of Dermatology and Venereology, Bangabandhu Sheikh Mujib Medical University, Dhaka

Correspondence Lubna Khondker, E-mail: lubnaderma@gmail.com 
streptococcal antigens have also been detected in the skin lesions of both guttate psoriasis (GP) and chronic plaque psoriasis (CPP), a subset of which recognize streptococcal cell wall antigens. ${ }^{8}$ It is possible that streptococci contain antigenic substances recognized by psoriatic T cells. ${ }^{9}$ Swerlick et $\mathrm{al}^{10}$ have demonstrated cross-reactivity between epidermal keratins and streptococci. There is a strong association between prior infection with streptococcus pyogenes (b-hemolytic streptococcus) and psoriasis which was proved by a history of an acute sore throat 1 to 3 weeks before the eruption and bacteriological (culture of throat swabs) and serological (ASO titer) evidence of recent streptococcal infection. ${ }^{11,12}$ Currently there is no cure for psoriasis and treatment options produce variable responses, partly, because disease pathogenesis is not completely understood. ${ }^{13}$

\section{Materials and Methods}

This cross sectional study was carried out in the department of Dermatology \& Venereology, Bangabandhu Sheikh Mujib Medical University (BSMMU), Dhaka from February to October 2012. Patients of both sexes and all ages with plaque type of psoriasis, diagnosed clinically and histopathologicaly, attending in department of Dermatology \& Venereology, BSMMU, Dhaka were selected as cases and age matched nonpsoriatic indoor and outdoor patients were included as controls. Consecutive type of nonprobability sampling technique was followed in this study. Controls were non-psoriasis patients (age matched within \pm 5 years with case) attending in inpatient and outpatient department of Dermatology \& Venereology, BSMMU. Patients on systemic anti-psoriatic drugs and/or systemic antibiotics currently and minimum within previous 15 days from interviewing were excluded. Informed written consent was taken from all the study subjects.

\section{Study procedure}

Patients with skin lesions suggestive of psoriasis attending in inpatient and outpatient department of Dermatology and Venereology, BSMMU were interviewed. Then these possible cases were examined clinically and advised for skin biopsy for histopathology and microbiological examination of throat swabs (culture and sensitivity) and serological test for antistreptococcal antibody (anti-streptolysin O [ASO] titer). Particulars of the subjects and history were recorded in a structured data sheet. After confirming psoriasis clinically and histopathologicaly and receiving both throat swab culture and ASO titer reports, possible cases were considered finally as confirmed cases. Clinically diagnosed psoriasis cases, but not confirmed histopathologically were excluded from the study. Thus total 47 plaque psoriasis patients of all ages and both sexes were recruited as cases finally. Recruited plaque psoriasis patients were allocated to one of three groups: 1) Guttate flare of chronic psoriasis $(n=14) ; 2)$ Chronic plaque psoriasis (including scalp psoriasis) $(n=26) ; 3)$ Sudden exacerbation/deterioration of chronic plaque psoriasis $(n=7)$.

Throat swab specimens were collected with sterile cotton swab (15 cm long) and venous blood was drawn with sterile syringe. Both the specimens were put into separate sterile test tubes for sending to the laboratories of Microbiology and Immunology departments of BSMMU as early as possible. Collected throat swabs were inoculated on blood agar media and incubated at $37^{0} \mathrm{C}$ for 48 hours. The pathogenic organism was identified by gross colony morphology and types of hemolysis (clear zone of hemolysis around the colony beta hemolysis or turbid halo with greenish zone around colony - alpha hemolysis) on culture media. b-hemolysis indicated growth of streptococcus pyogenes. ASO titers were measured with BN ProSpec, SIEMENS, USA. ASO titer $>200 \mathrm{IU} / \mathrm{mL}$ or greater (upper limit of normal value, $200 \mathrm{IU} / \mathrm{mL}$ ) was considered as evidence of recent streptococcal infections. Data were collected from preformed data sheets and bacteriological and serological reports. Statistical analysis was done by using SPSS (version 16.0). Statistical significance was set at 5\% level and confidence interval at $95 \%$ level.

\section{Results}

In this study mean age was $38 \pm 4.7$ years in cases and $29 \pm 5.4$ years in controls. There was no significant difference in age between two groups. Table I shows distribution of subjects according to age. 
Table I: Distribution of subjects according to age

\begin{tabular}{|c|c|c|c|}
\hline \multirow[t]{2}{*}{ Age group (yrs) } & Cases $(n=47)$ & Controls $(n=39)$ & $\mathrm{p}$ value \\
\hline & Frequency (\%) & Frequency (\%) & \\
\hline $10-19$ & $13(27.66)$ & $9(23.07)$ & \\
\hline $20-29$ & $14(29.79)$ & $14(35.89)$ & \\
\hline $30-39$ & $12(25.53)$ & $9(23.07)$ & \\
\hline $40-49$ & $4(8.51)$ & $4(10.25)$ & \\
\hline $50-59$ & $4(8.51)$ & $3(7.69)$ & \\
\hline Mean \pm SD & $38 \pm 4.7$ & $29 \pm 5.4>0.05$ & ns \\
\hline
\end{tabular}

$\mathrm{p}$ value obtained from $\mathrm{t}$ test; $\mathrm{ns}=$ Nonsignificant

Fig 1 shows the distribution of different types of plaque psoriasis in cases. Majority (55\%) were diagnosed as chronic plaque psoriasis.

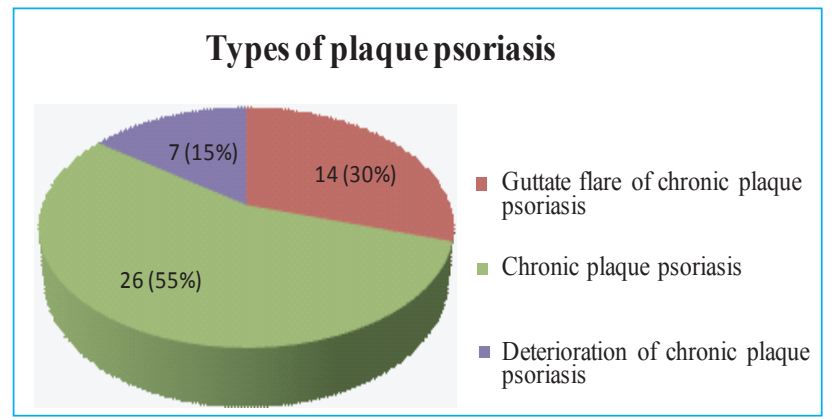

Fig 1. Types of plaque psoriasis in cases

Fig 2 shows the distribution of guttate flare of chronic plaque psoriasis patients according to their history of sore throat within 1 to 4 weeks period prior to the appearance of skin lesions. It shows that $64.2 \%$ of guttate flare of chronic plaque psoriasis patients gave a positive history of sore throat. Other types of psoriatic patients did not give history of sore throat.

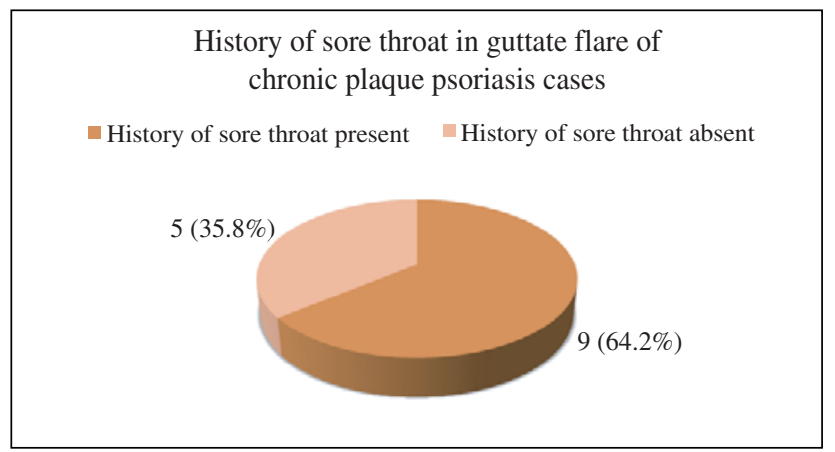

Fig 2. History of sore throat in guttate flare of chronic plaque psoriasis prior to the appearance of skin lesions
ASO titer status in cases and controls is shown in Table II. ASO titer was raised (>200 IU/mL) in $28(59.57 \%)$ patients and $7(17.90 \%)$ nonpsoriatic controls. The difference between two groups was significant $(p<0.05)$.

Table II: Comparison of ASO titer status between cases and controls

\begin{tabular}{|c|c|c|c|c|c|}
\hline \multirow[t]{2}{*}{ ASO titer status } & \multicolumn{2}{|c|}{ Cases $(n=47)$} & \multicolumn{2}{|c|}{ Controls $(n=39)$} & \multirow[t]{2}{*}{$\mathrm{p}$ value } \\
\hline & Frequenc & ercentage & Frequency & Percentage & \\
\hline $\begin{array}{l}\text { Raised } \\
(>200 \mathrm{IU} / \mathrm{mL})\end{array}$ & 28 & 59.57 & 7 & 17.90 & \\
\hline $\begin{array}{l}\text { Within normal range } \\
(<200 \mathrm{IU} / \mathrm{mL})\end{array}$ & 19 & 40.43 & 32 & 82.10 & $<0.05^{*}$ \\
\hline Total & 47 & 100.00 & 39 & 100.00 & \\
\hline
\end{tabular}

$\mathrm{p}$ value obtained from chi-square test; *Significant

Table III: Level of ASO titer in different types of plaque psoriatic patients $(n=47)$

\begin{tabular}{|l|c|c|}
\hline Types of psoriasis & $\begin{array}{l}\text { ASO titer } \\
\text { Raised }(>200 \\
\text { IU/mL) }\end{array}$ & $\begin{array}{c}\text { ASO titer } \\
\text { within normal } \\
\text { limit }(<200 \mathrm{IU} / \mathrm{mL})\end{array}$ \\
\hline $\begin{array}{l}\text { Guttate flare of chronic } \\
\text { plaque psoriasis }\end{array}$ & $11(78.6 \%)$ & $3(21.4 \%)$ \\
\hline $\begin{array}{l}\text { Chronic plaque } \\
\text { psoriasis }\end{array}$ & $12(46.2 \%)$ & $14(53.8 \%)$ \\
\hline $\begin{array}{l}\text { Deterioration of } \\
\text { chronic plaque psoriasis }\end{array}$ & $5(71.4 \%)$ & $2(28.6 \%)$ \\
\hline Total & $28(60 \%)$ & $19(40 \%)$
\end{tabular}

Table IV shows comparison of throat swab culture reports between cases and controls. Table V shows the throat swab culture results in different types of psoriasis. In total streptococcus pyogenes was isolated from 12 (25.50\%) plaque psoriasis patients and 4 (10.25\%) controls. There was no significant difference in culture results of two groups ( $\mathrm{p}>0.05$ ).

Table IV: Comparison of throat swab culture reports between cases and controls

\begin{tabular}{|l|c|c|c|}
\hline Organisms & $\begin{array}{c}\text { Cases } \\
(\mathrm{n}=47)\end{array}$ & $\begin{array}{c}\text { Controls } \\
(\mathrm{n}=39)\end{array}$ & $\mathrm{p}$ value \\
\hline Streptococcus pyogenes & 12 & 4 & \\
\hline Normal flora & 33 & 34 & $>0.05$ \\
\hline Staph/Klebsiella spp & 2 & 1 & \\
\hline
\end{tabular}

$\mathrm{p}$ value obtained from $\mathrm{c}^{2}$ test $\left(\mathrm{df}=2, \mathrm{c}^{2}\right.$ value $\left.=3.59\right)$

${ }^{*} \mathrm{p}$ value is $>0.05$; Not significant 
Table V: Results of throat swab culture in different types of psoriasis $(n=47)$

\begin{tabular}{|c|c|c|c|}
\hline \multirow[t]{2}{*}{ Types of psoriasis } & \multicolumn{3}{|c|}{ Culture results } \\
\hline & S. pyoger & Normal flora & $\begin{array}{c}\text { Staph/ } \\
\text { Klebsiella spp }\end{array}$ \\
\hline $\begin{array}{l}\text { Guttate flare of } \\
\text { chronic plaque } \\
\text { psoriasis }\end{array}$ & $4(21.4 \%)$ & $10(78.6 \%)$ & 0 \\
\hline $\begin{array}{l}\text { Chronic plaque } \\
\text { psoriasis }\end{array}$ & $5(15.3 \%)$ & $20(80.8 \%)$ & $1(3.8 \%)^{\dagger}$ \\
\hline $\begin{array}{l}\text { Deterioration of } \\
\text { chronic plaque } \\
\text { psoriasis }\end{array}$ & $3(42.8 \%)$ & $3(42.8 \%)$ & $1(14.2 \%)^{\dagger}$ \\
\hline
\end{tabular}

\section{Discussion}

Among the 47 cases in this study 26 (55\%) subjects were of chronic plaque psoriasis, 14 (30\%) patients were with guttate flare of chronic plaque psoriasis and $7(15 \%)$ patients were with deterioration of chronic plaque psoriasis. Similar study was conducted by Telfar et al with 111 patients with psoriasis, where they found $60 \%$ cases as chronic plaque psoriasis. ${ }^{12}$

This study shows that $64.2 \%$ patients of guttate flare of chronic plaque psoriasis gave a positive history of sore throat and their history of sore throat was within 1 to 4 weeks period prior to the appearance of skin lesions. ASO titer was raised in $59.6 \%$ of plaque psoriasis patients and it was $17.9 \%$ in controls. Difference of ASO titer status in chronic plaque psoriasis was significant in comparison with respective controls ( $p$ value $<0.05$ ). Serologic evidence of recent streptococcal infections (raised ASO titer) was found in 18 (56\%) of 32 patients with $31 \%$ having a history of sore throat 1 to 3 weeks before the appearance of rash and $17(85 \%)$ of 20 patients with AGP (acute guttate psoriasis) in studies reported by Mukherjee et al. ${ }^{14}$ In a cross sectional study with 111 patients, ASO titer was raised in $43 \%$ cases in total, $58 \%$ in AGP and $26 \%$ in guttate exacerbation of chronic plaque psoriasis. ${ }^{12}$ In a prospective study, Gudjohnson et al described ASO titer status raised 10 times than their controls in chronic plaque psoriasis. ${ }^{4}$ All these studies showed significant differences ( $\mathrm{p}$ value $<0.05$ ) with their respective controls. The outcomes of above mentioned studies were similar to our findings.
In this study, Streptococcus pyogenes was isolated from $12(25.5 \%)$ plaque type of psoriasis patients and 4 (10.26\%) controls. There was no significant difference in culture result of two groups ( $\mathrm{p}>0.05)$. Confirmation of streptococcal infections in psoriasis might be difficult because patients were mostly seen in the convalescent phase when antibiotics had already been taken and throat swab cultures were more likely to be negative. Streptococcus pyogenes was isolated from $26 \%$ in AGP, $13 \%$ in guttate flare of chronic plaque psoriasis, $14 \%$ in chronic plaque psoriasis and $6 \%$ in total controls in a study on 111 psoriasis patients by Telfar et al. ${ }^{12}$ In another study Naqqash et al isolated streptococcus pyogenes in $34 \%$ cases in chronic plaque psoriasis and $97 \%$ in AGP. ${ }^{6}$ Gudjohnson et al showed Streptococcus pyogenes positive in throat swab culture in $9.1 \%$ in chronic plaque psoriasis versus $0.9 \%$ in controls $(p<0.05) .{ }^{4}$ Keeping these studies in mind and the possible association documented in our study, between Streptococcus pyogenes and plaque psoriasis, in terms of evidence of recent throat infections (raised ASO titer and positive throat swab culture), it is important to search for and eliminate microbial infections in the treatment of plaque type of psoriasis.

From this study it can be concluded that streptococcal throat infections can provocate plaque psoriasis. However, more studies are needed with greater numbers of patients to determine risk associations of psoriasis with S. pyogenes.

\section{Limitation}

Limitation of this study was difficulty in confirmation of streptococcal infections in psoriasis because patients are often seen in the convalescent phase when antibiotics have already been taken and throat swab cultures are more likely to be negative.

\section{References}

1. Griffiths CEM, Camp RDR, Barker JNWN. Psoriasis. In: Rook's textbook of dermatology. $7^{\text {th }}$ edn. Masachusetts: Blackwell Publisher, 2004: 35-69.

2. Neimann AL, Porter SB, Gelfand JM. The epidemiology of psoriasis. Expert revision of dermatology 2006; 1(1): 63-75.

3. Terui T, Ozawa M, Tagami H. Role of neutrophils in induction of acute inflammation in T-cell-mediated immune dermatosis, psoriasis: a neutrophil-associated inflammationboosting loop. Experimental Dermatology 2000; 9(1): 1-10.

4. Gudjhonson JE, Thorarisson AM, Sigurgeirsson B, Kristinsson $\mathrm{KG}$, Valdimarsson H. Streptococcal throat infections and 
exacerbation of chronic plaque psoriasis: a prospective study. British Journal of Dermatology 2003; 149: 530-534.

5. Leung DY, Walsh P, Giorno R, Norris DA. A potential role for superantigens in the pathogenesis of psoriasis. J Invest Dermatol 1993; 100: 225-228.

6. Naqqash S, Uddeen T, Naqqash SH, Butt AK. Family history of psoriasis and recent infectious disease are risk factors for the first episode of acute guttate psoriasis. Journal of Pakistan Association of Dermatologists 2004; 14: 124-129.

7. McFadden J, Valdimarsson H, Fry L. Cross-reactivity between streptococcal M surface antigen and human skin. British Journal of Dermatology 1991; 125: 443-447.

8. Korotky NG, Peslyak MY. Psoriasis as a consequence of incorporation of beta-streptococci into the microbiocenosis of highly permeable intestines. Vestn Dermatol Venerol 2005; 1: 9-18.

9. Baker BS, Powel AV, Malkani AK. Altered cell-mediated immunity to group A haemolytic streptococcal antigens in chronic plaque psoriasis. British Journal of Dermatology 1991; 125: 38-42.

10. Swerlick RA, Cunningham MW, Hall NK. Monoclonal antibodies cross-reactive with group A streptococci and normal and psoriatic human skin. J Invest Dermatol 1986; 87: $367-371$.

11. Villeda GG, Santamaria CLC, Perez LR. Recognition of Streptococcus pyogenes and skin autoantigens in guttate psoriasis. Arch Med Res 1998; 29: 143-148.

12. Telfer NR, Chalmers RJG, Whale K, Colman G. The role of Streptococcal infection in the initiation of guttate psoriasis. Arch Dermatol 1992; 128: 39-42.

13. Naldi L, Griffiths CEM. Traditional therapies in the management of moderate to severe chronic plaque psoriasis: an assessment of the benefits and risks. British Journal of Dermatology 2005; 152(4): 597-615.

14. Mukherjee SK. Streptococcal infection as trigger for psoriasis. Contemporary Pediatrics 2011; 2(1): 207-215. 